\title{
Distances to nearby galaxies in Sculptor ${ }^{\star}$
}

\author{
I. D. Karachentsev ${ }^{1}$, E. K. Grebel ${ }^{2}$, M. E. Sharina ${ }^{1,10}$, A. E. Dolphin ${ }^{3}$, D. Geisler ${ }^{4}$, P. Guhathakurta ${ }^{5}$, P. W. Hodge ${ }^{6}$, \\ V. E. Karachentseva ${ }^{7}$, A. Sarajedini ${ }^{8}$, and P. Seitzer ${ }^{9}$
}

1 Special Astrophysical Observatory, Russian Academy of Sciences, N. Arkhyz, KChR, 369167, Russia

2 Max-Planck-Institut für Astronomie, Königstuhl 17, 69117 Heidelberg, Germany

3 Kitt Peak National Observatory, National Optical Astronomy Observatories, PO Box 26732, Tucson, AZ 85726, USA

${ }^{4}$ Departamento de Física, Grupo de Astronomía, Universidad de Concepción, Casilla 160-C, Concepción, Chile

5 UCO/Lick Observatory, University of California at Santa Cruz, Santa Cruz, CA 95064, USA

6 Department of Astronomy, University of Washington, Box 351580, Seattle, WA 98195, USA

7 Astronomical Observatory of Kiev University, 04053, Observatorna 3, Kiev, Ukraine

8 Department of Astronomy, University of Florida, Gainesville, FL 32611, USA

9 Department of Astronomy, University of Michigan, 830 Dennison Building, Ann Arbor, MI 48109, USA

10 Isaac Newton Institute, Chile, SAO Branch

Accepted 9 December 2002 / Accepted 31 January 2003

\begin{abstract}
We present an analysis of Hubble Space Telescope/WFPC2 images of nine nearby galaxies in Sculptor. We derive their distances from the luminosity of the tip of the red giant branch stars with a typical accuracy of $\sim 12 \%$. Their distances are 4.21 Mpc (Sc 22), 4.92 Mpc (DDO 226), $3.94 \mathrm{Mpc}$ (NGC 253), 3.40 Mpc (KDG 2), $3.34 \mathrm{Mpc}$ (DDO 6), $3.42 \mathrm{Mpc}$ (ESO 540-030), 4.43 Mpc (ESO 245-05), 4.27 Mpc (UGCA 442), and 3.91 Mpc (NGC 7793). The galaxies are concentrated in several spatially separated loose groups around NGC 300, NGC 253, and NGC 7793. The Sculptor galaxy complex together with the CVn I cloud and the Local Group form a $10 \mathrm{Mpc}$ filament, apparently driven by the free Hubble flow.
\end{abstract}

Key words. galaxies: dwarf - galaxies: distances and redshifts - galaxies: kinematics and dynamics

\section{Introduction}

The association of five bright spiral galaxies in the Sculptor constellation, NGC 55, NGC 247, NGC 253, NGC 300, and NGC 7793, has been considered by many authors as the galaxy group closest to the Local Group (LG), at a distance of $\sim 2 \mathrm{Mpc}$. Over the past decades, a number of dwarf irregular galaxies in this area have been found by van den Bergh (1959: DDO 6, DDO 226), Nilsen (1974: UGCA 438, UGCA 442), and Lauberts (1982: ESO sky survey objects). Special searches for fainter irregular (dIrr) and spheroidal (dSph) dwarf galaxies in the Sculptor group were undertaken by Côté et al. (1997), Karachentseva \& Karachentsev (1998, 2000), and Jerjen et al. $(1998,2000)$. In their paper entitled, "Discovery of numerous dwarf galaxies in the two nearest groups of galaxies", Côté et al. (1997) reported on the discovery of five new dwarf members of the Sculptor group: Sc 2, Sc 18, Sc 22, Sc 24, and Sc 42. However, all of them, except Sc 22, were found then to be

Send offprint requests to: M. E. Sharina, e-mail: sme@luna.sao.ru

* Based on observations made with the NASA/ESA Hubble Space Telescope. The Space Telescope Science Institute is operated by the Association of Universities for Research in Astronomy, Inc. under NASA contract NAS 5-26555. background galaxies (see the CCD images in Whiting et al. 2002), whose H I velocities were confused with galactic high velocity clouds in the velocity range from +60 to $+160 \mathrm{~km} \mathrm{~s}^{-1}$. Recent H I surveys of the Sculptor region by Staveley-Smith et al. (1998), Barnes et al. (2001), de Blok et al. (2002) have not yet led to the discovery of new dwarf members of the group. Jerjen et al. (1998) measured distances to five dwarf galaxies in Sculptor from fluctuations of their surface brightness and showed that the so-called "Sculptor group" turns out to be a loose filament of galaxies extended along a line of sight over $\sim 5 \mathrm{Mpc}$.

In this paper we present new distance measurements for nine galaxies in the Sculptor region, derived from their tip of the red giant branch (TRGB). Together with earlier published TRGB distances to four other galaxies (Karachentsev et al. 2000, 2002) and the distance to NGC 300 from Cepheids (Freedman et al. 1992), this gives us a basis for a more detailed study of the structure of the Sculptor complex.

\section{WFPC2 photometry}

Images of nine galaxies were obtained with the Wide Field and Planetary Camera (WFPC2) aboard the Hubble Space 


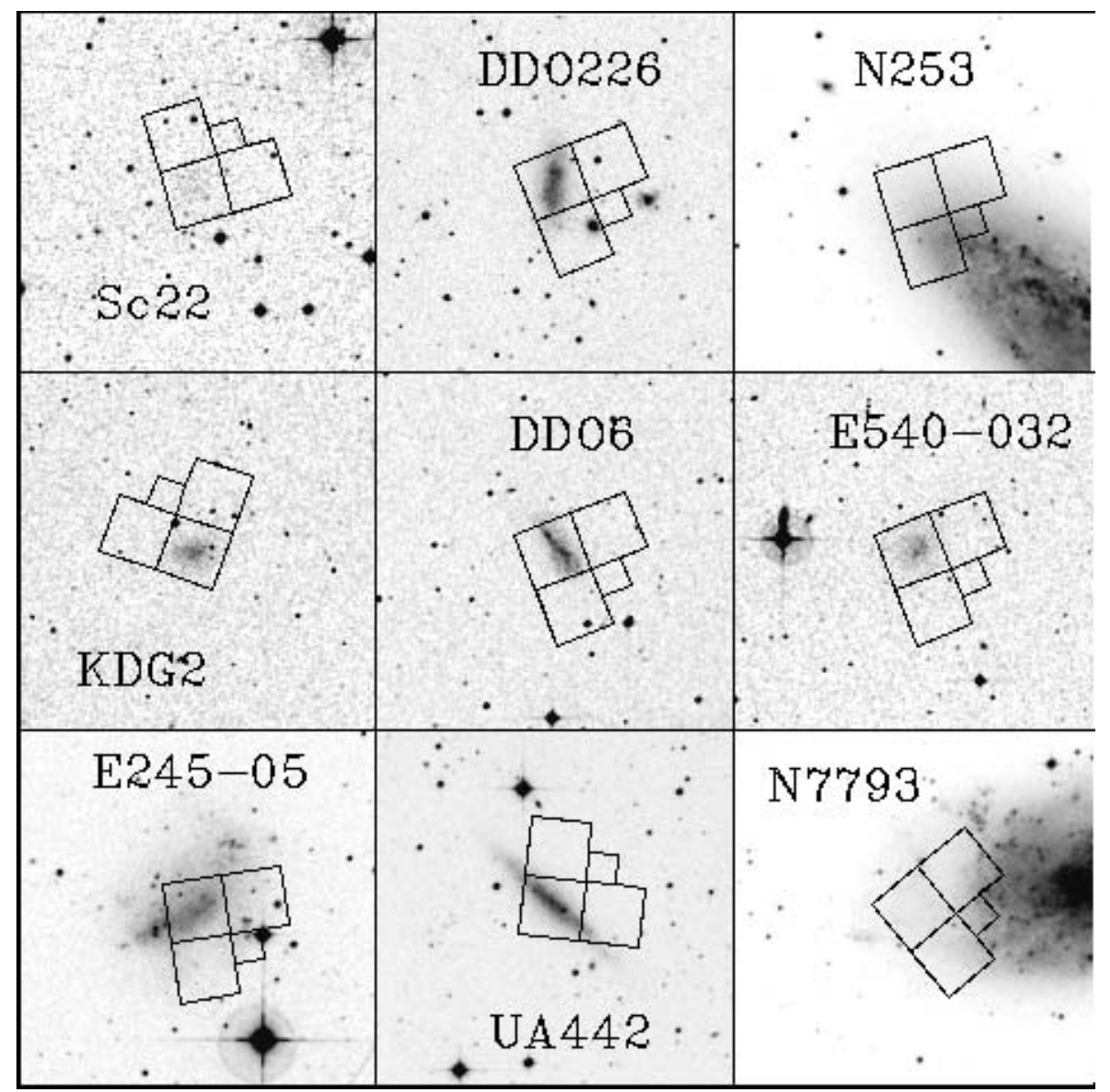

Fig. 1. Digital Sky Survey images of 9 nearby galaxies in Sculptor. The field size is $8^{\prime}$, North is up and East is left. The HST WFPC2 footprints are superimposed.

Telescope (HST) between August 24, 1999 and June 28, 2001 as part of our HST snapshot survey of nearby galaxy candidates (Seitzer et al. 1999; Grebel et al. 2000). The galaxies were observed with 600-second exposures taken in the F606W and F814W filters for each object. Digital Sky Survey (DSS) images of these galaxies are shown in Fig. 1 with the HST WFPC2 footprints superimposed. The field size of the red DSSII images is $8^{\prime}$. Small galaxies were usually centered on the WF3 chip, but for some bright objects the WFPC2 position was shifted towards the galaxy periphery to decrease stellar crowding. The WFPC2 images of the galaxies are presented in the upper panels of Fig. 2, where both filters are combined.

For photometric measurements we used the HSTphot stellar photometry package developed by Dolphin (2000a). The package has been optimized for the undersampled conditions present in the WFPC2 to work in crowded fields. After removing cosmic rays, simultaneous photometry was performed on the F606W and F814W frames using multiphot, with corrections to an aperture with a radius of 0.'5. Charge-transfer efficiency (CTE) corrections and calibrations were then applied, which are based on the Dolphin (2000b) formulae, producing $V, I$ photometry for all stars detected in both images. Additionally, stars with a signal-to-noise ratio $S / N<3,|\chi|>$ 2.0 , or $\mid$ sharpness $\mid>0.4$ in each exposure were eliminated from the final photometry list. The uncertainty of the photometric zero point is estimated to be within 0.05 (Dolphin 2000b).

\section{TRGB distances to nine galaxies in Sculptor}

The tip of red giant branch (TRGB) method provides an efficient tool to measure galaxy distances. The TRGB distances agree with those given by the Cepheid period-luminosity relation to within $5 \%$. As shown by Lee et al. (1993), the TRGB is relatively independent of age and metallicity. In the $I$ band the TRGB for low-mass stars is found to be stable within $\sim 0$. 1 (Salaris \& Cassisi 1997; Udalski et al. 2001) for metallicities, $[\mathrm{Fe} / \mathrm{H}]$, encompassing the entire range from -2.1 to $-0.7 \mathrm{dex}$ found in galactic globular clusters. According to Da Costa \& Armandroff (1990), for metal-poor systems the TRGB is located at $M_{I}=-4$ m 05 . Ferrarese et al. (2000) calibrated the zero point of the TRGB from galaxies with Cepheid distances and estimated $M_{I}=-4.06 \pm 0.07$ (random) \pm 0.13 (systematic). A new TRGB calibration, $M_{I}=-4 .{ }^{\mathrm{m}} 04 \pm 0.12$, was made by Bellazzini et al. (2001) based on photometry and on the distance estimate from a detached eclipsing binary in the galactic globular cluster $\omega$ Centauri. For this paper we use $M_{I}=-4$. 05 . The lower left panels of Fig. 2 show $I,(V-I)$ color-magnitude diagrams (CMDs) for the nine observed galaxies. 

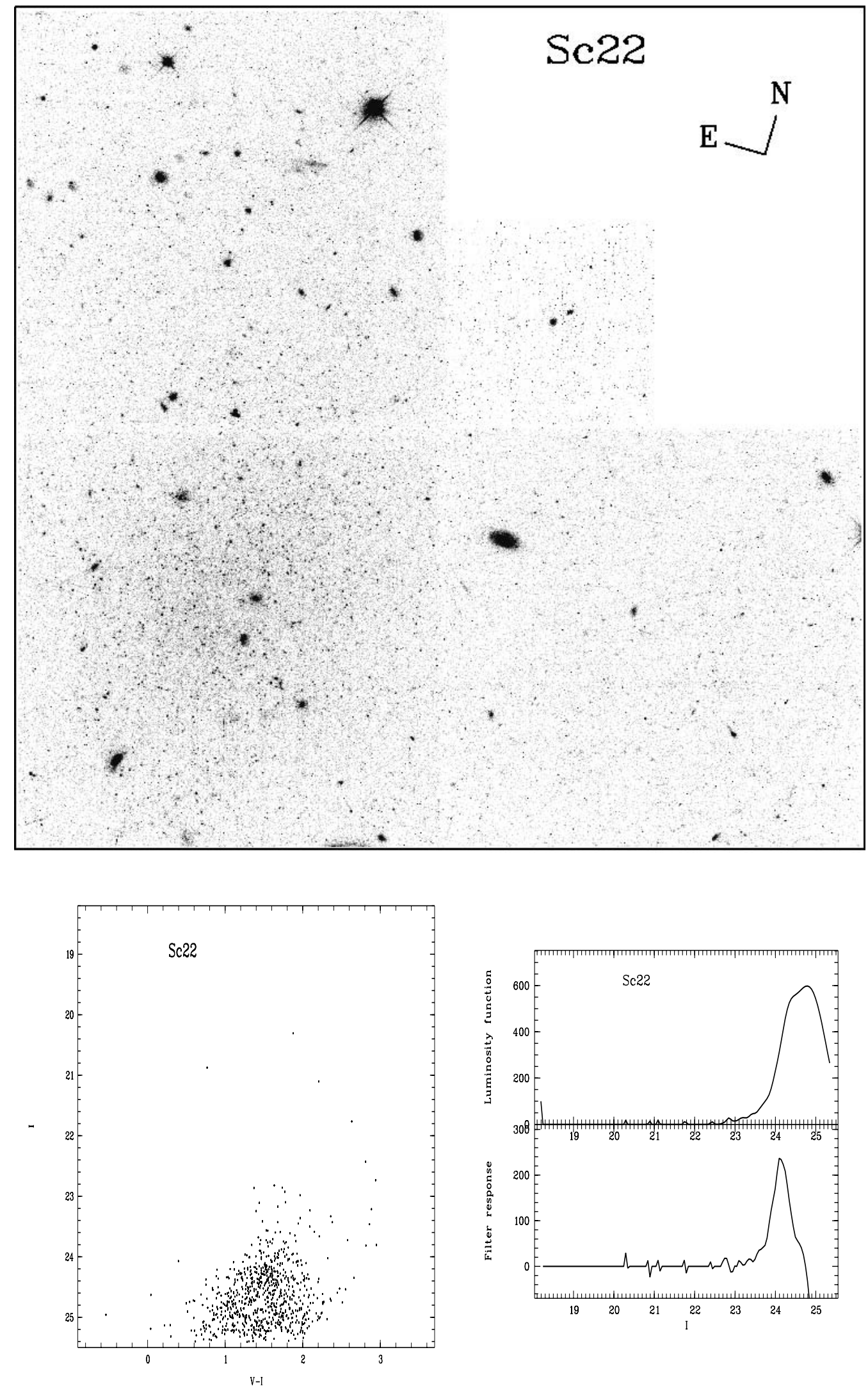

Fig. 2. Top: WFPC2 images of nine galaxies: Sc 22, DDO 226, NGC 253, KDG 2, DDO 6, ESO 540-032, ESO 245-05, UGCA 442, and NGC 7793 produced by combining the two 600s exposures obtained through the F606W and F814W filters. The arrows point to the North and the East. Bottom left: The color-magnitude diagrams from the WFPC2 data for the nine galaxies in Sculptor. Bottom right: The Gaussian-smoothed $I$-band luminosity function restricted to red stars (top), and the output of an edge-detection filter applied to the luminosity function for the nine galaxies. 

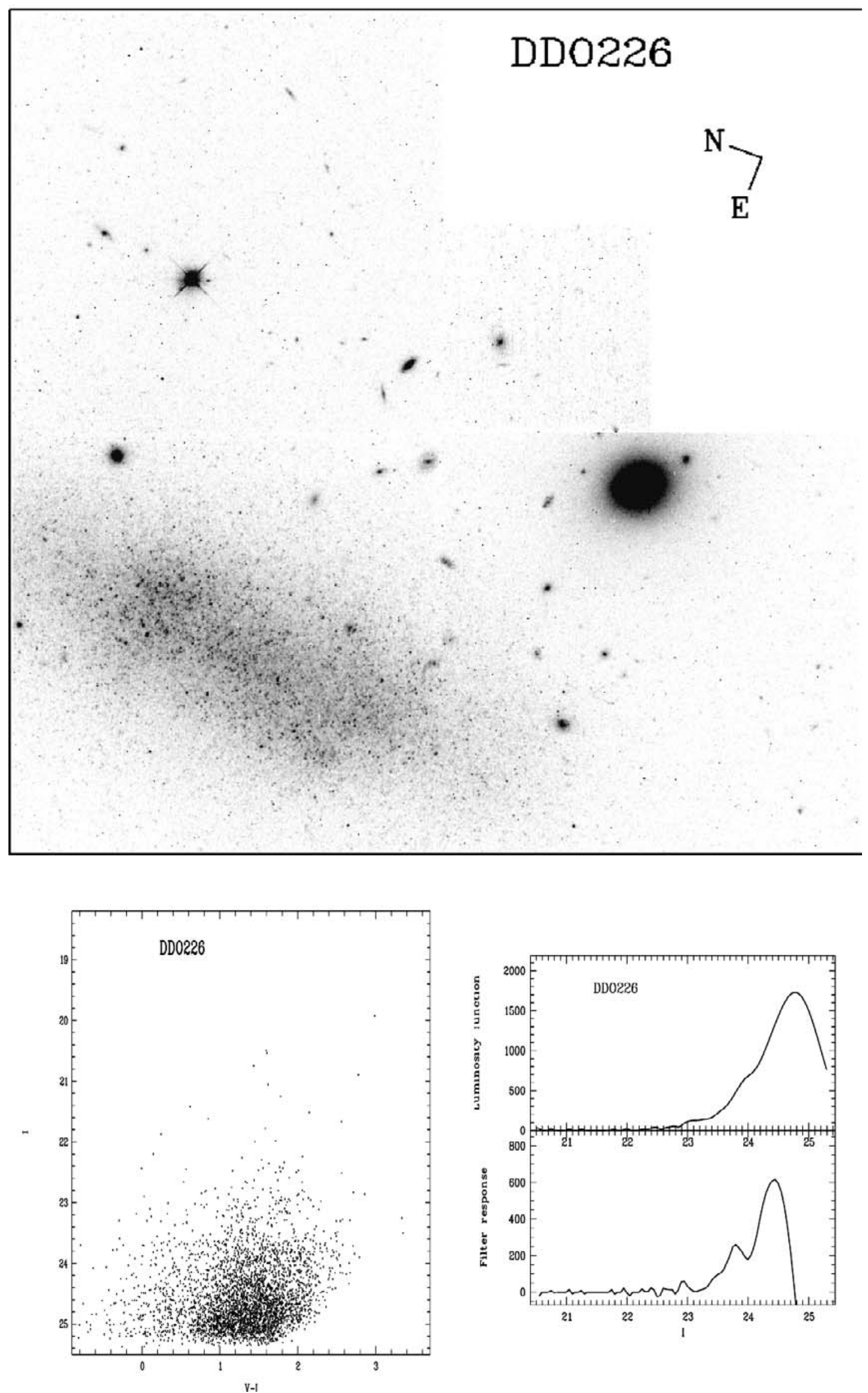

Fig. 2. continued. 

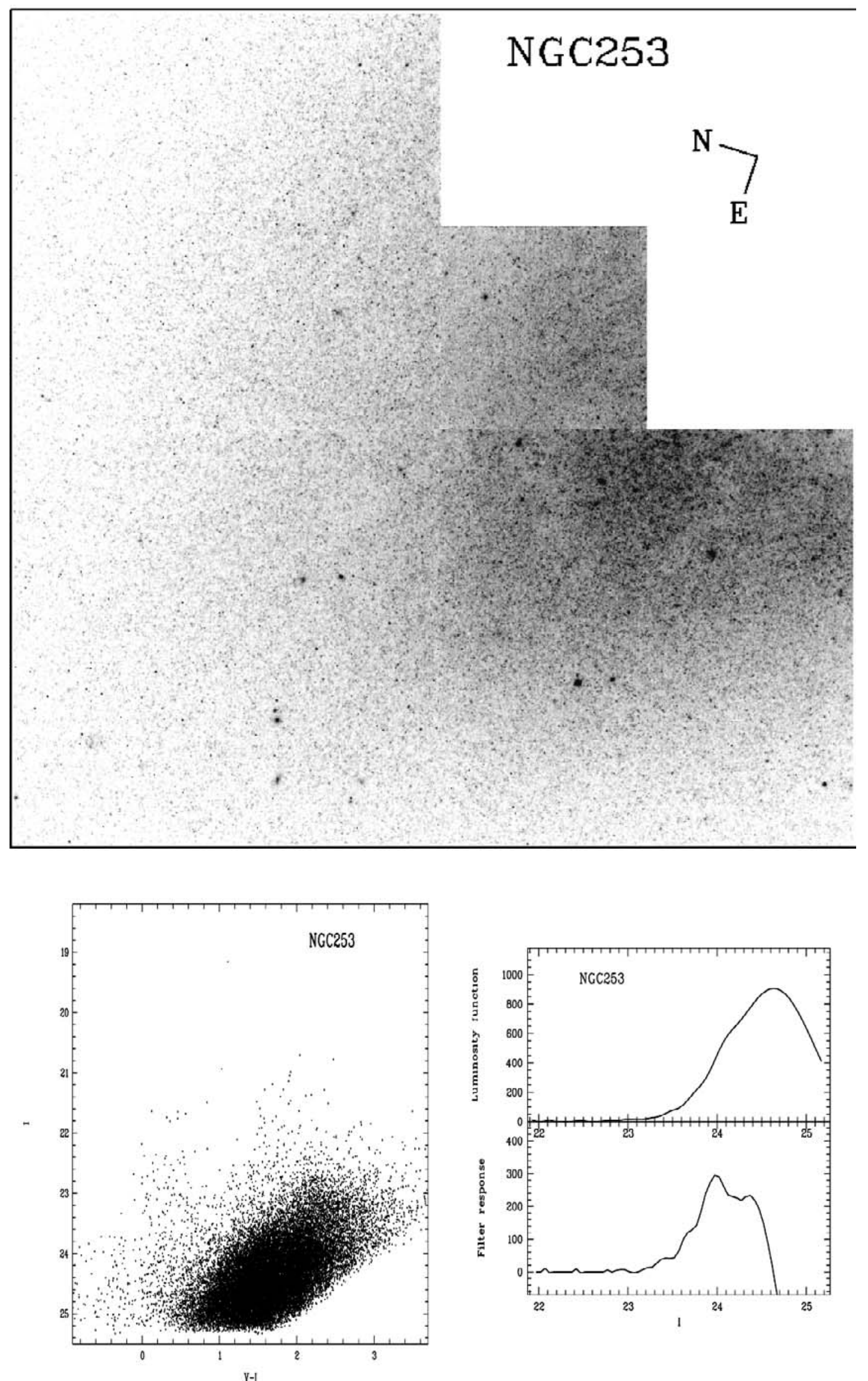

Fig. 2. continued. 

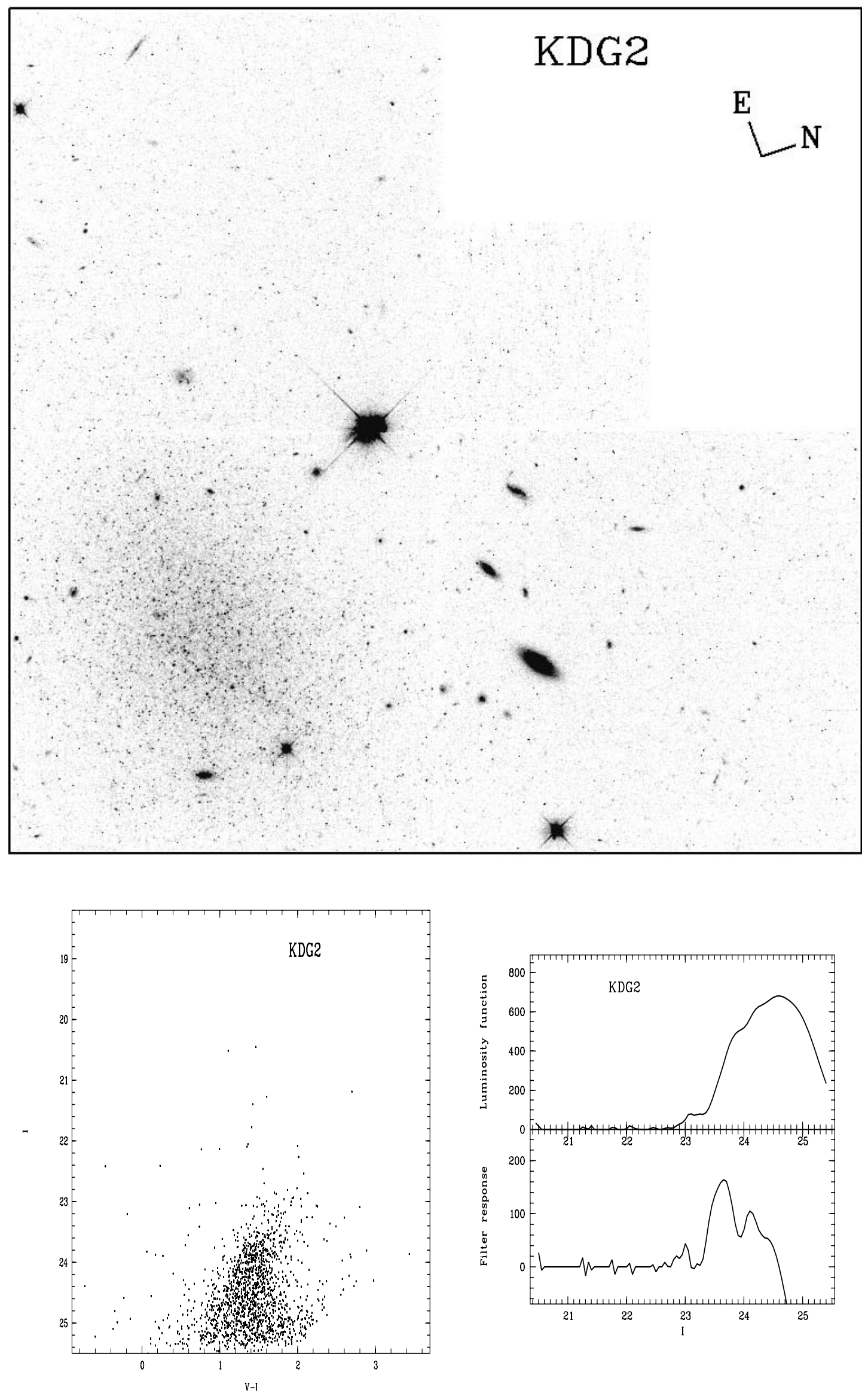

Fig. 2. continued. 

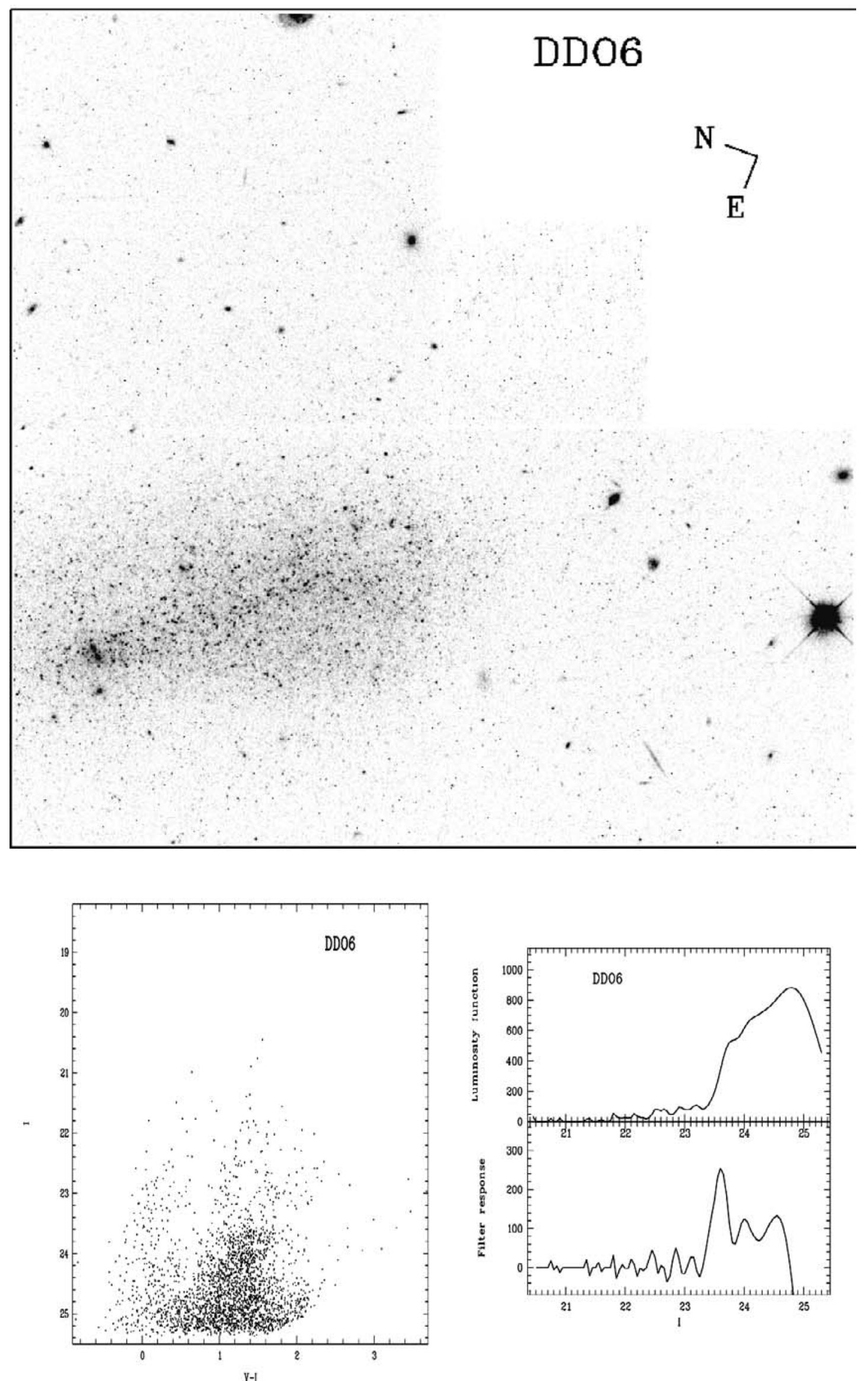

Fig. 2. continued. 

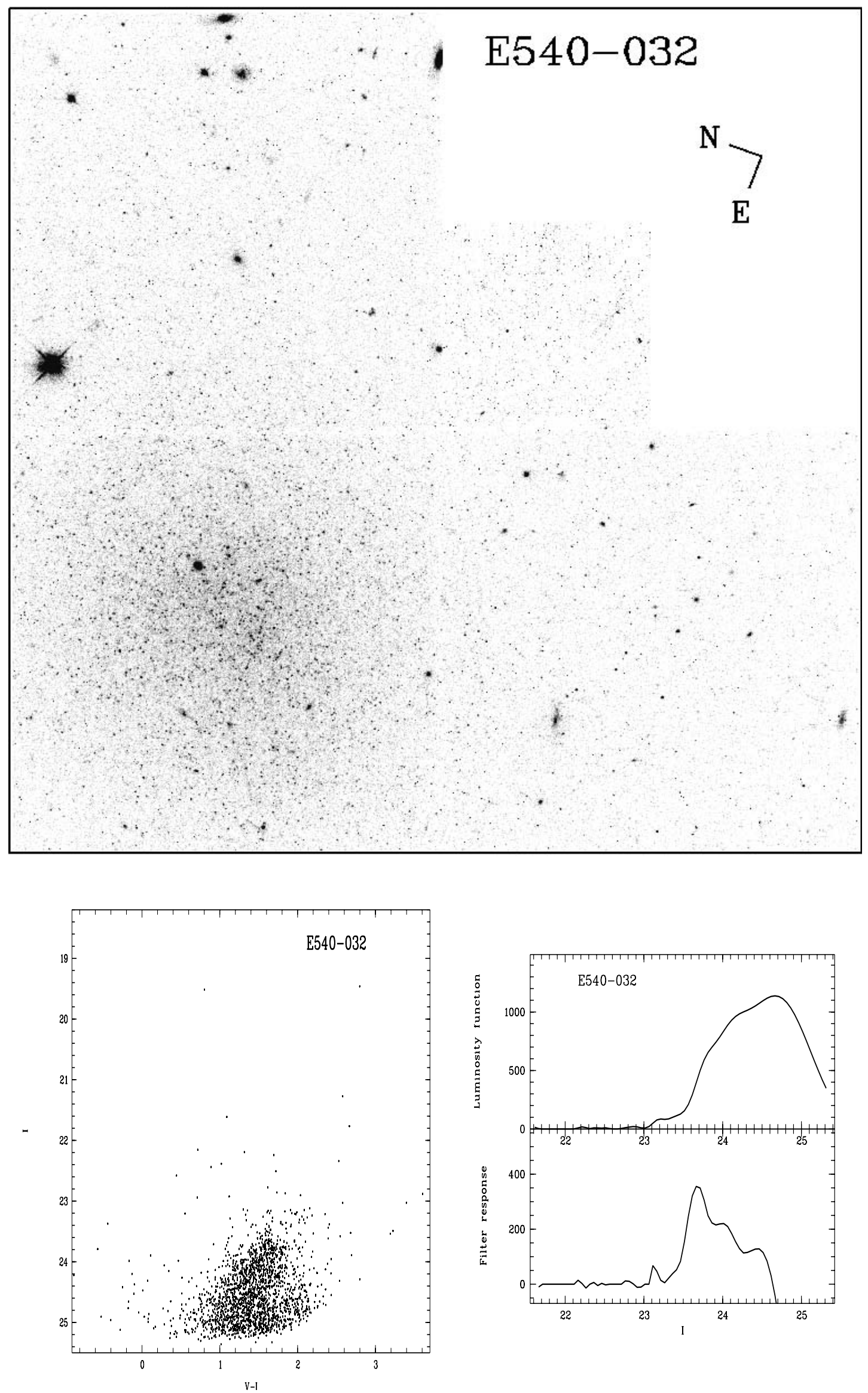

Fig. 2. continued. 

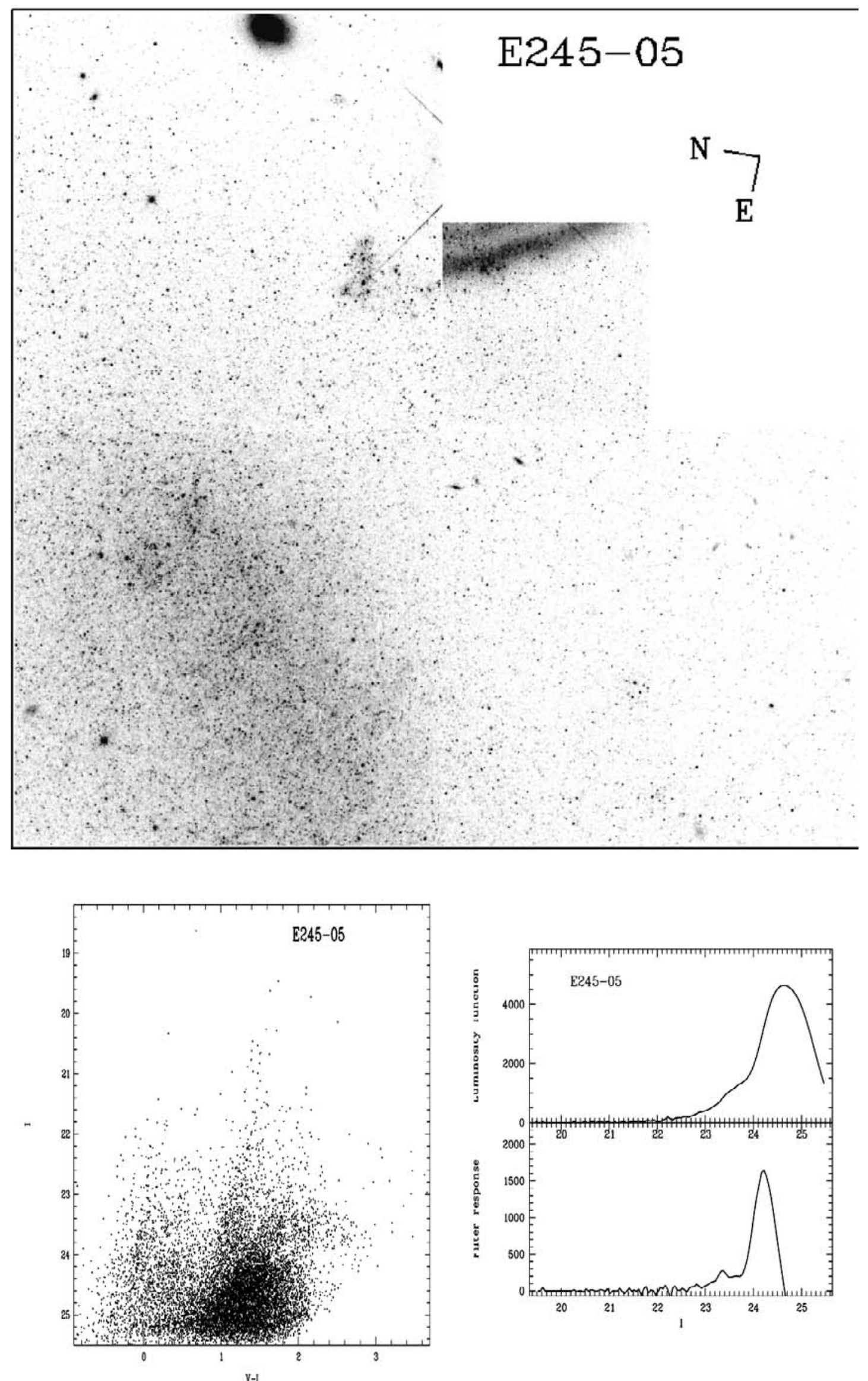

Fig. 2. continued. 

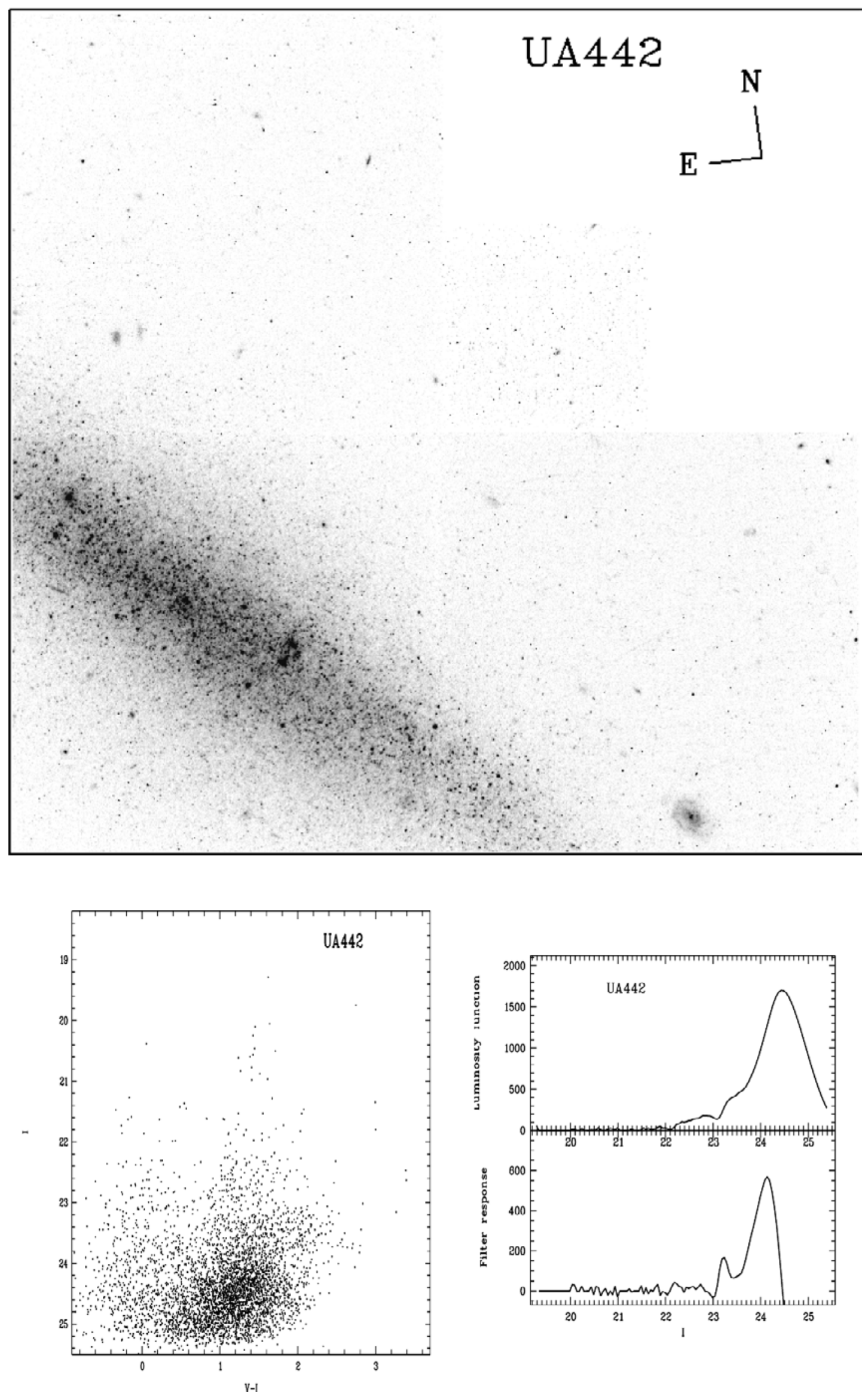

Fig. 2. continued. 

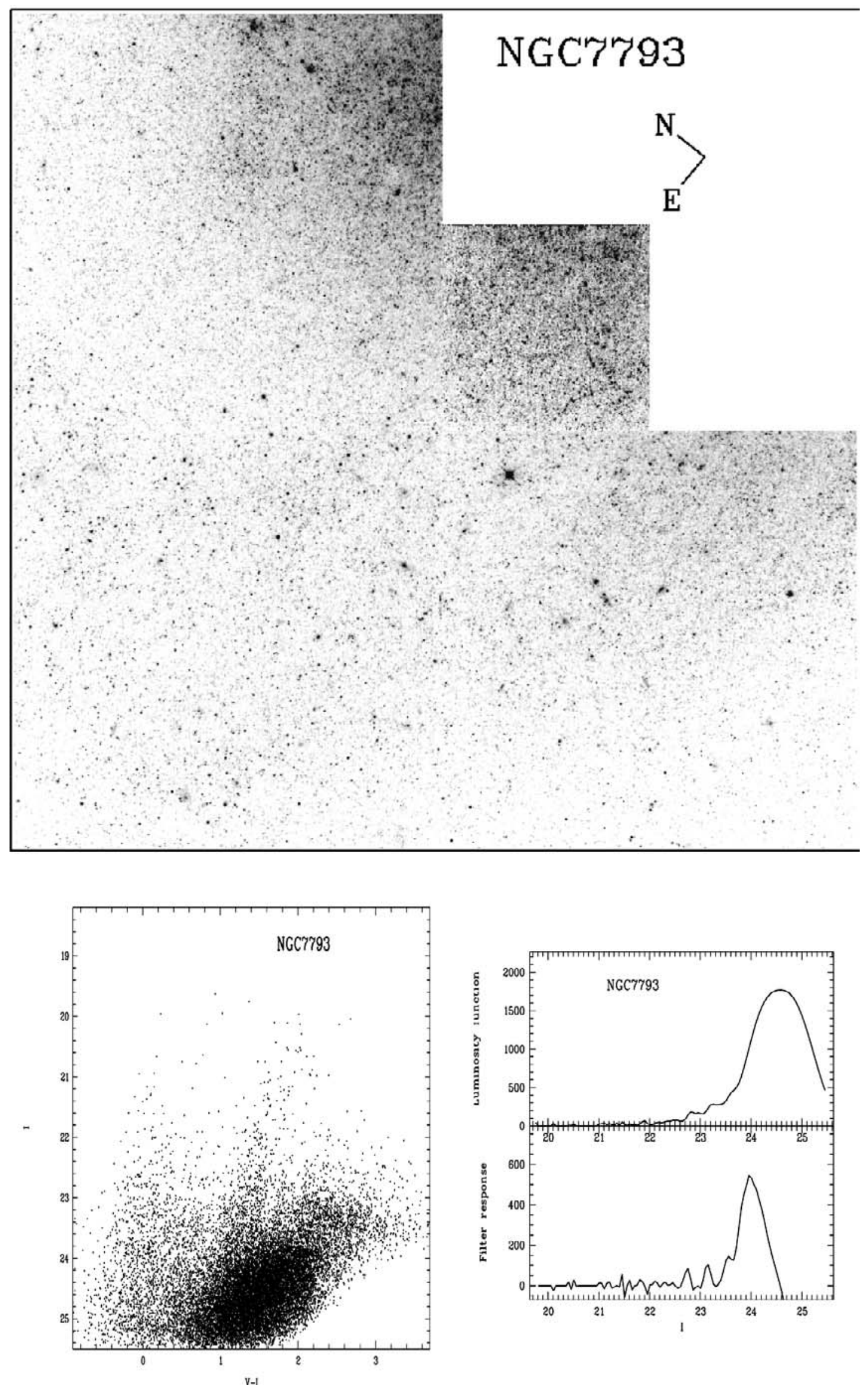

Fig. 2. continued. 
Table 1. New distances to galaxies in Sculptor region.

\begin{tabular}{|c|c|c|c|c|c|c|c|c|c|}
\hline Name & RA (B1950) Dec & $\begin{array}{r}a \\
b / a \\
\end{array}$ & $\begin{array}{l}B_{T} \\
A_{b} \\
\end{array}$ & $T$ & $\begin{array}{r}V_{h} \\
V_{\mathrm{LG}} \\
\end{array}$ & $I(T R G B)$ & $(m-M)_{0}$ & $D_{\mathrm{MW}}$ & $\begin{array}{r}(V-I)_{-3.5} \\
{[\mathrm{Fe} / \mathrm{H}]}\end{array}$ \\
\hline \multirow[t]{2}{*}{$\mathrm{Sc} 22$} & $002121.0-245855$ & 0.9 & 17.73 & -3 & & 24.10 & 28.12 & 4.21 & 1.40 \\
\hline & & .78 & 0.06 & & & 0.21 & 0.23 & 0.43 & -1.51 \\
\hline \multirow[t]{2}{*}{ DDO226 } & $004035.0-223127$ & 2.2 & 14.36 & 10 & 357 & 24.44 & 28.46 & 4.92 & 1.28 \\
\hline & & .36 & 0.07 & & 408 & 0.24 & 0.26 & 0.58 & -1.96 \\
\hline \multirow[t]{2}{*}{$\mathrm{N} 253$} & $004506.9-253354$ & 26.7 & 7.92 & 5 & 241 & 23.97 & 27.98 & 3.94 & 1.54 \\
\hline & & .22 & 0.08 & & 274 & 0.19 & 0.21 & 0.37 & -1.12 \\
\hline KDG2 & $004651.9-182048$ & 1.2 & 16.37 & -1 & & 23.65 & 27.66 & 3.40 & 1.37 \\
\hline E540-030 & & .92 & 0.10 & & & 0.20 & 0.22 & 0.34 & -1.61 \\
\hline \multirow[t]{2}{*}{ DDO6 } & $004721.0-211718$ & 1.7 & 15.19 & 10 & 295 & 23.60 & 27.62 & 3.34 & 1.25 \\
\hline & & .41 & 0.07 & & 348 & 0.13 & 0.16 & 0.24 & -2.08 \\
\hline E540-032 & $004756.0-201044$ & 1.3 & 16.44 & -3 & & 23.66 & 27.67 & 3.42 & 1.42 \\
\hline FG24 & & .92 & 0.09 & & & 0.14 & 0.17 & 0.27 & -1.45 \\
\hline E245-05 & $014257.9-435054$ & 3.8 & 12.73 & 10 & 394 & 24.22 & 28.23 & 4.43 & 1.25 \\
\hline P6430 & & 0.89 & 0.07 & & 308 & 0.21 & 0.23 & 0.45 & -2.08 \\
\hline \multirow[t]{2}{*}{ UA442 } & $234109.0-321412$ & 6.4 & 13.58 & 9 & 267 & 24.13 & 28.15 & 4.27 & 1.18 \\
\hline & & .14 & 0.07 & & 299 & 0.26 & 0.27 & 0.52 & -2.40 \\
\hline \multirow[t]{2}{*}{ N7793 } & $235515.0-325206$ & 9.3 & 9.70 & 7 & 229 & 23.95 & 27.96 & 3.91 & 1.50 \\
\hline & & .68 & 0.08 & & 252 & 0.22 & 0.24 & 0.41 & -1.22 \\
\hline
\end{tabular}

We determined the TRGB using a Gaussian-smoothed $I$-band luminosity function (LF) for red stars with colors $V-I$ within \pm 0 . 5 of the mean $\langle V-I\rangle$ expected for red giant branch stars. Following Sakai et al. (1996), we applied a Sobel edgedetection filter. The position of the TRGB was identified with the peak in the filter response function. The resulting LFs and the Sobel-filtered LFs are shown in the lower right corners of Fig. 2. The results are summarized in Table 1. There we list: (1) galaxy name; (2) equatorial coordinates of the galaxy center; (3) galaxy major diameter and axial ratio; (4) apparent integrated blue magnitude from the NASA Extragalactic Database (NED) and galactic extinction in the $B$-band from Schlegel et al. 1998; (5) morphological type in de Vaucouleurs' notation; (6) heliocentric radial velocity and radial velocity with respect to the LG centroid (Karachentsev \& Makarov 1996); (7) position of the TRGB and its uncertainty as derived with the Sobel filter; (9) true distance modulus with its uncertainty, which takes into account the uncertainty in the TRGB, as well as uncertainties of the HST photometry zero point $\left(\sim 0{ }^{\mathrm{m}} 05\right)$, the aperture corrections $\left(\sim 0 .{ }^{\mathrm{m}} 05\right)$, and the crowding effects $(\sim 0.06)$ added quadratically; the uncertainties in the extinction and reddening are taken to be $10 \%$ of their values from Schlegel et al. (1998); (for more details on the total budget of internal and external systematic errors for the TRGB method see Mendez et al. 2002); and (9) linear distance in Mpc and its uncertainty.
Given the distance moduli of the galaxies, we can estimate their mean metallicity from the mean color of the TRGB measured at an absolute magnitude $M_{I}=-3.5$, as recommended by Da Costa \& Armandroff (1990). Based on a Gaussian fit to the color distribution of the giant stars in a corresponding $I$-magnitude interval $(-3.5 \pm 0.3)$, we derived their mean colors, $(V-I)_{-3.5}$, which lie in the range of 1.18 mag to $1.54 \mathrm{mag}$ after correction for galactic reddening. Following the relation of Lee et al. (1993), this provides us with mean metallicities $-1.1 \mathrm{dex}>\langle[\mathrm{Fe} / \mathrm{H}]\rangle>-2.4 \mathrm{dex}$, listed in the last column of Table 1. With a typical statistical scatter of the mean color $(\sim 0.05)$, and uncertainties of the HST photometry zero point we expect the uncertainty in metallicity to be about 0.3 dex. Therefore within the measurement accuracy the metallicity of the galaxies satisfy the required limitation, $[\mathrm{Fe} / \mathrm{H}]<-0.7 \mathrm{dex}$. Below, some individual properties of the galaxies are briefly discussed.

Sc 22. This dwarf spheroidal galaxy of very low surface brightness was discovered by Côté et al. (1997). Surface photometry of Sc 22 was carried out by Jerjen et al. (1998, 2000), who determined its integrated apparent magnitude, $B_{T}=17.73 \mathrm{mag}$, integrated color, $(B-R)_{T}=0.79 \mathrm{mag}$, and central surface brightness, $25.8^{m} / \square^{\prime \prime}$ in the $B$ band. Using the method of surface brightness fluctuations (SBF), they estimated the distance to Sc 22 to be $2.67 \pm 0.16 \mathrm{Mpc}$. 
The galaxy was not detected in the Hi line by Côté et al. (1997), neither was it detected in the "blind" HIPASS survey by Staveley-Smith et al. (1998) and Barnes et al. (2001). The color-magnitude diagram of Sc 22 (see Fig. 2) is populated predominantly by red stars. The application of the Sobel filter yields $I($ TRGB $)=24 .{ }^{\mathrm{m}} 10 \pm 0.21$, which corresponds to a linear distance of $4.21 \pm 0.43 \mathrm{Mpc}$. This is much larger than the SBF distance.

DDO 226 = IC 1574 = UGCA 9. This dIrr galaxy has a relatively high radial velocity, $V_{\mathrm{LG}}=408 \mathrm{~km} \mathrm{~s}^{-1}$. The CMD of DDO 226 shows blue and red stellar populations. Due to large number of red stars, which probably belong to the asymptotic giant branch (AGB), the location of the TRGB is determined with a low level of confidence. According to the data in Fig. 2, I(TRGB) $=24 . \mathrm{m}^{\mathrm{m}} 44 \pm 0.24$, which yields a linear distance of $4.92 \pm 0.58 \mathrm{Mpc}$.

NGC 253. NGC 253 is the brightest galaxy in the Sculptor group. With a size of $27^{\prime} \times 6^{\prime}$, NGC 253 extends far beyond the WFPC2 field. Surprisingly, this prominent Sc galaxy has so far no reliable distance estimate, apart from a rough estimate of $D=2.77 \mathrm{Mpc}$ derived by Puche \& Carignan (1988) from the Tully-Fisher relation. In our HST observations the WFPC2 was pointed to the NE part of the galaxy, where stellar the crowding is lower. The HST photometry detects a total of about 27000 stars, mostly of red colors. The TRGB is located at $I=23.97 \pm 0$. m 19 , corresponding to a distance of $3.94 \pm$ $0.37 \mathrm{Mpc}$. With this distance, the resulting absolute integrated magnitude of NGC 253, $M_{B}=-20.14$ mag, turns out to be comparable with the absolute magnitudes of the Milky Way and other brightest members of the nearest groups.

KDG 2 = ESO 540-030 = KK 09. This dSph galaxy of low surface brightness was found by Karachentseva (1968) and then selected as the Local Volume member candidate by Karachentseva \& Karachentsev (1998). According to the surface photometry carried out by Jerjen et al. (1998, 2000), KDG 2 has an integrated magnitude of $B_{T}=16.37 \mathrm{mag}$, an integrated color of $(B-R)_{T}=0.83 \mathrm{mag}$, and a central surface brightness of $24.1^{\mathrm{m}} / \square^{\prime \prime}$ in the $B$-band. Jerjen et al. (1998) determined its distance via SBF to be $3.19 \pm 0.13 \mathrm{Mpc}$. The HST photometry detects about 1900 predominantly red stars. We derived the TRGB to be located at $23.65 \pm 0.20$, which corresponds to $D=3.40 \pm 0.34 \mathrm{Mpc}$, in good agreement with the previous estimate. Apart from red stars, we also found a number of blue stars, which occupy the central part of the galaxy. Probably, KDG 2 is not a dSph galaxy, but belongs to a transition dSph/dIrr type, like LGS-3 and Antlia. Huchtmeier et al. (2000) did not detect it in the H I line.

DDO 6 = UGCA 15 = ESO 540-031. This dIrr galaxy of drop-like shape has a radial velocity of $V_{\mathrm{LG}}=348 \mathrm{~km} \mathrm{~s}^{-1}$. In Fig. 2 the CMD shows the presence of mixed blue and red populations, in particular, a prominent population of RGB stars. We determined $I$ (TRGB) to be $23^{\mathrm{m}} \cdot 62 \pm 0.16$, yielding $D=$ $3.34 \pm 0.24 \mathrm{Mpc}$.

ESO 540-032 = FG $24=$ KK 10. Like KDG 2, FG 24 (Feitzinger \& Galinski 1985) has a reddish color and a low surface brightness, typical of $\mathrm{dSph}$ or $\mathrm{dSph} / \mathrm{dIrr}$ galaxies. The galaxy was not detected in $\mathrm{HI}$ by Huchtmeier et al. (2000). Surface photometry of FG 24, performed by
Jerjen et al. (1998, 2000), yields an integrated magnitude of $B_{T}=16.44 \mathrm{mag}$, an integrated color $(B-R)_{T}=1.08 \mathrm{mag}$, and a central surface brightness $24.5^{\mathrm{m}} / \square^{\prime \prime}$ in the $B$-band. Most of the stars detected by us in FG 24 (see Fig. 2) are likely RGB stars, although some faint blue stars are present in the galaxy's central part. The TRGB I-band magnitude derived by us, $23 \mathrm{~m} .67 \pm 0$. 17 , corresponds to a distance of $3.42 \pm 0.27 \mathrm{Mpc}$, which exceeds the distance $2.21 \pm 0.14 \mathrm{Mpc}$ estimated by Jerjen et al. (1998) from SBF, but agrees excellently with the new distance estimate, $3.4 \pm 0.2 \mathrm{Mpc}$, obtained by Jerjen $\&$ Rejkuba (2001) from the TRGB.

ESO 245-005. This irregular galaxy of Magellanic type has an angular dimension of 3.' $\times 3.4$ and a radial velocity of $V_{\mathrm{LG}}=308 \mathrm{~km} \mathrm{~s}^{-1}$. In the WFPC 2 image of its central barlike part we detected about 11000 blue and red stars. We determined the $I$-band TRGB to be at $24.22 \pm 0.21$, yielding a distance of $4.43 \pm 0.45 \mathrm{Mpc}$.

UGCA 442 = ESO 471-06. This is an edge-on galaxy of Im type with a radial velocity $V_{\mathrm{LG}}=299 \mathrm{~km} \mathrm{~s}^{-1}$ and angular dimension 6.4 × 0.9, much larger than the WFPC2 field. The galaxy seems to be well resolved into stars. Our HST photometry reveals about 5600 stars, both blue and red ones. The derived TRGB, $I=24 . \mathrm{m} 13 \pm 0$. 26 , corresponds to a distance of $4.27 \pm 0.52 \mathrm{Mpc}$.

NGC 7793. This is a bright spiral galaxy with a dimension of $9.3 \times 6.3$ and with a radial velocity $V_{\mathrm{LG}}=$ $252 \mathrm{~km} \mathrm{~s}^{-1}$. The WFPC2 was pointed at its eastern side. There are about 22000 stars detected by us in both the filters. The CMD shows a mixture of young and old stellar populations. The TRGB magnitude, 23 m $95 \pm 0.22$, yields a galaxy distance of $3.91 \pm 0.41 \mathrm{Mpc}$, in satisfactory agreement with the distance $3.27 \pm 0.08 \mathrm{Mpc}$ derived by Puche \& Carignan (1988) from the Tully-Fischer relation.

\section{Structure and kinematics of the Sculptor group}

To study the 3D structure of the Sculptor complex, we collected the most complete sample of data on all known nearby galaxies situated in this sky region. Table 2 presents the following characteristics of the 21 galaxies of our sample: (1) galaxy name; (2) equatorial (upper line), and Supergalactic (lower line) coordinates; (3) major angular diameter in arcmin and apparent axial ratio; (4) apparent integrated magnitude from NED and galactic extinction from Schlegel et al. (1998) in the $B$-band; (5) morphological type; (6) heliocentric radial velocity in $\mathrm{km} \mathrm{s}^{-1}$ and velocity in the Local Group rest frame (Karachentsev \& Makarov 1996); (7) the H I line width (in $\mathrm{km} \mathrm{s}^{-1}$ ) at the $50 \%$ level of the maximum from LEDA (Paturel et al. 1996) or HIPASS, corrected for galaxy inclination (upper line), and absolute magnitude of the galaxy, corrected for galactic extinction (lower line); (8) distance to the galaxy in Mpc from the Milky Way (upper line), and from the Local Group centroid (lower line), respectively. The last column gives the method used for distance measurement ("Cep" from Cepheids, "RGB" - from TRGB, "SBF" - from surface brightness fluctuations, and "TF" - from the Tully-Fisher relation), and the distance data reference. 
Table 2. List of 21 nearby galaxies in the Sculptor group region.

\begin{tabular}{|c|c|c|c|c|c|c|c|c|c|}
\hline Name & $\begin{array}{c}\text { RA (B1950) Dec } \\
S G L \quad S G B\end{array}$ & $\begin{array}{r}a \\
b / a\end{array}$ & $\begin{array}{l}B_{T} \\
A_{b}\end{array}$ & $T$ & $\begin{array}{r}V_{h} \\
V_{\mathrm{LG}} \\
\end{array}$ & $\begin{array}{r}W_{50} c \\
M_{b}\end{array}$ & $\begin{array}{r}D_{\mathrm{MW}} \\
D_{\mathrm{LG}}\end{array}$ & $\begin{array}{r}\mathrm{d} V_{\mathrm{p}} \\
R\end{array}$ & Notes \\
\hline E349-031 & $000540.9-345124$ & 1.1 & 15.48 & 10 & 207 & 35 & 4.1 & 44 & $\mathrm{TF}$ \\
\hline SDIG & $260.18+0.40$ & .82 & 0.05 & & 216 & -12.65 & 4.02 & 0.90 & present paper \\
\hline \multirow[t]{2}{*}{ N55 } & 001238.0-392954 & 32.4 & 8.84 & 9 & 129 & 175 & 1.8 & 170 & $\mathrm{TF}$ \\
\hline & $256.25-2.36$ & .17 & 0.06 & & 111 & -17.50 & 1.78 & 2.26 & present paper \\
\hline N59 & $001253.0-214318$ & 2.7 & 13.12 & -3 & 361 & & 5.3 & 164 & SBF \\
\hline KK2 & $273.13+3.15$ & .48 & 0.09 & & 431 & -15.59 & 5.12 & 1.62 & Jerjen et al. (1998) \\
\hline E410-005 & $001300.3-322728$ & 1.3 & 14.90 & -1 & & & 1.92 & & RGB \\
\hline KK3 & $262.95-0.26$ & .77 & 0.06 & & & -11.58 & 1.85 & & Karachentsev et al. (2000) \\
\hline \multirow[t]{2}{*}{$\operatorname{Sc} 22$} & $002121.0-245855$ & 0.9 & 17.73 & -3 & & & 4.21 & & RGB \\
\hline & $270.62+0.31$ & .78 & 0.06 & & & -10.45 & 4.05 & & present paper \\
\hline \multirow[t]{2}{*}{ E294-010 } & $002406.2-420756$ & 1.1 & 15.60 & -3 & 117 & & 1.92 & 195 & RGB \\
\hline & $254.37-5.27$ & .64 & 0.02 & & 81 & -10.84 & 1.85 & 2.06 & Karachentsev et al. (2002) \\
\hline \multirow[t]{2}{*}{ DDO226 } & $004035.0-223127$ & 2.2 & 14.36 & 10 & 357 & 51 & 4.92 & 134 & RGB \\
\hline & $274.23-3.21$ & .36 & 0.07 & & 408 & -14.17 & 4.74 & 1.01 & present paper \\
\hline \multirow[t]{2}{*}{ N247 } & $004439.6-210158$ & 21.4 & 9.86 & 7 & 160 & 222 & 4.09 & 19 & $\mathrm{TF}$ \\
\hline & 275.92-3.73 & .32 & 0.08 & & 215 & -18.28 & 3.90 & 0.36 & present paper \\
\hline \multirow[t]{2}{*}{$\mathrm{N} 253$} & $004506.9-253354$ & 26.7 & 7.92 & 5 & 241 & 420 & 3.94 & 0 & RGB \\
\hline & 271.57-5.01 & .22 & 0.08 & & 274 & -20.14 & 3.79 & 0 & present paper \\
\hline KDG2 & $004651.9-182048$ & 1.2 & 16.37 & -1 & & & 3.40 & & RGB \\
\hline E540-030 & $278.65-3.52$ & .92 & 0.10 & & & -11.39 & 3.20 & & present paper \\
\hline \multirow[t]{2}{*}{ DDO6 } & $004721.0-211718$ & 1.7 & 15.19 & 10 & 295 & 24 & 3.34 & -53 & RGB \\
\hline & $275.84-4.40$ & .41 & 0.07 & & 348 & -12.50 & 3.16 & 0.55 & present paper \\
\hline E540-032 & $004756.0-201044$ & 1.3 & 16.44 & -3 & & & 3.42 & & RGB \\
\hline FG24 & $276.95-4.24$ & .92 & 0.09 & & & -11.32 & 3.23 & & present paper \\
\hline \multirow[t]{2}{*}{ N300 } & $005231.8-375712$ & 21.9 & 8.95 & 7 & 144 & 212 & 2.15 & 163 & Cep \\
\hline & $259.81-9.50$ & .71 & 0.06 & & 114 & -17.77 & 2.11 & 1.78 & Freedman et al. (1992) \\
\hline \multirow[t]{2}{*}{ N625 } & $013254.9-414130$ & 6.4 & 11.59 & 9 & 405 & 94 & 2.7: & 23 & $\mathrm{TF}$ \\
\hline & 257.26-17.74 & .28 & 0.07 & & 335 & -15.64 & 2.69 & 1.65: & present paper \\
\hline \multirow[t]{2}{*}{ E245-05 } & $014257.9-435054$ & 3.8 & 12.73 & 9 & 394 & & 4.43 & 116 & RGB \\
\hline & $255.14-19.74$ & .89 & 0.07 & & 308 & -15.57 & 4.30 & 1.67 & present paper \\
\hline I5152 & $215926.6-513214$ & 5.2 & 11.06 & 10 & 124 & 116 & 2.07 & 212 & RGB \\
\hline E237-27 & $234.23+11.53$ & .62 & 0.11 & & 75 & -15.63 & 2.18 & 2.63 & Karachentsev et al. (2002) \\
\hline \multirow[t]{2}{*}{ KK258 } & $223756.3-310340$ & 1.6 & 17.36 & -3 & & & & & \\
\hline & $255.48+18.58$ & .50 & 0.06 & & & & & & \\
\hline \multirow[t]{2}{*}{ UA438 } & $232347.3-323957$ & 1.5 & 13.86 & 10 & 62 & 57 & 2.23 & 179 & RGB \\
\hline & $258.88+9.28$ & .80 & 0.06 & & 99 & -12.94 & 2.16 & 1.86 & Karachentsev et al. (2002) \\
\hline \multirow[t]{2}{*}{ UA442 } & $234109.0-321412$ & 6.4 & 13.58 & 9 & 267 & 94 & 4.27 & 82 & RGB \\
\hline & $260.78+6.12$ & .14 & 0.07 & & 299 & -14.64 & 4.01 & 1.15 & present paper \\
\hline \multirow[t]{2}{*}{ E149-003 } & $234925.5-525121$ & 2.2 & 15.0 & 10 & 577 & 56 & 6.4 & 293 & $\mathrm{TF}$ \\
\hline & $242.31-3.23$ & .18 & 0.06 & & 501 & -14.10 & 6.44 & 3.54 & present paper \\
\hline \multirow[t]{2}{*}{ N7793 } & $235515.0-325206$ & 9.3 & 9.70 & 7 & 229 & 237 & 3.91 & 60 & RGB \\
\hline & $261.30+3.12$ & .68 & 0.08 & & 252 & -18.34 & 3.82 & 0.90 & present paper \\
\hline
\end{tabular}




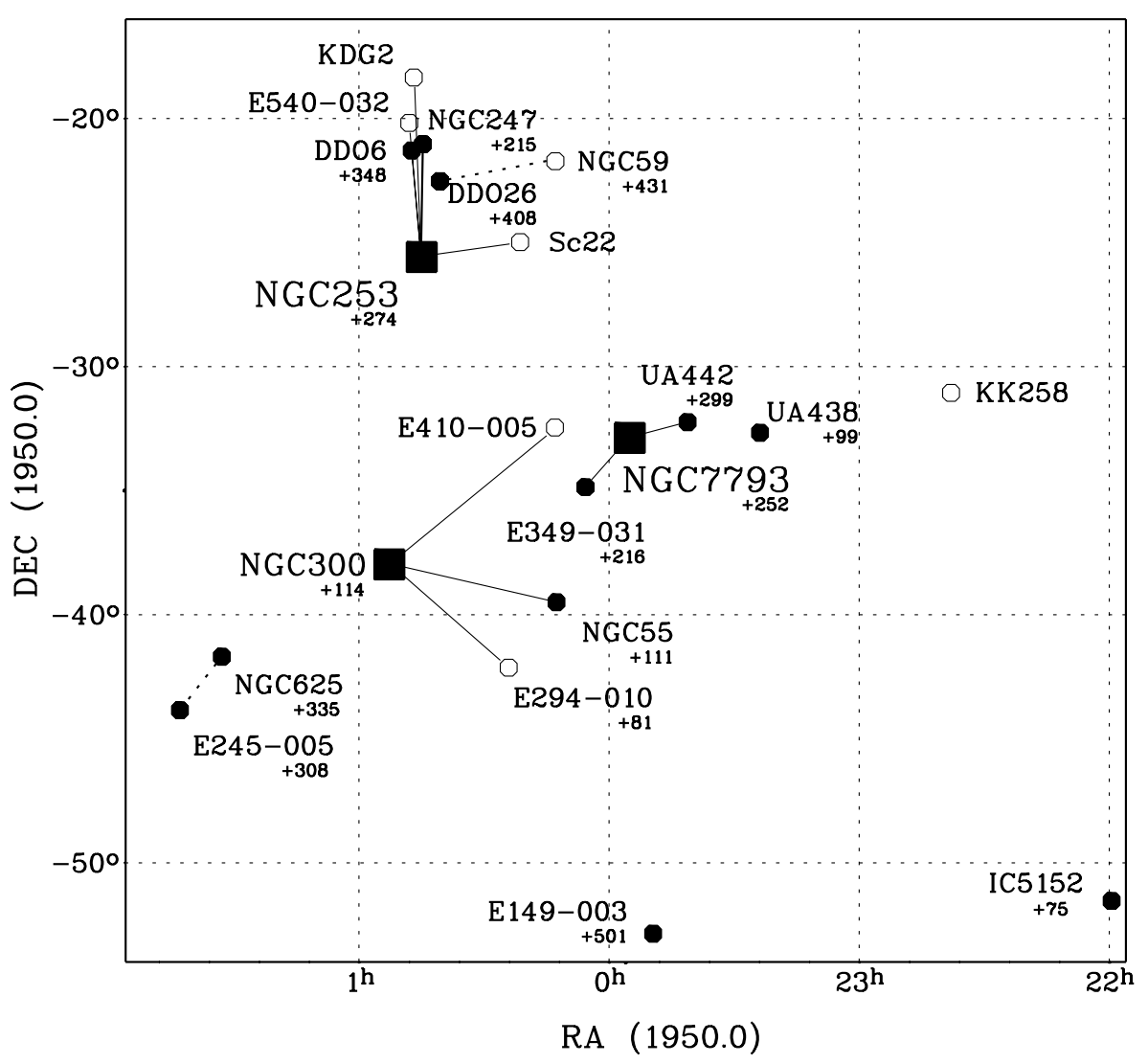

Fig. 3. Sky distribution of nearby galaxies in the direction of the Sculptor group. Filled and open circles indicate dwarf irregular and dwarf spheroidal galaxies, respectively. Large squares correspond to the most luminous galaxy in each group. Their probable companions are connected to the principal galaxies with straight lines. Radial velocities of the galaxies relative to the Local Group centroid are indicated by small numbers.

Apart from four dSph galaxies with known distances but unknown radial velocities (KK 3, Sc 22, KDG 2, and FG 24), we included in Table 2 also a low surface brightness galaxy KK 258 with unknown distance and velocity, which looks like a nearby semi-resolved system at CCD image derived by Whiting et al. (2002). In comparison with the TRGB distances, the distance estimates of Sc 22, KDG 2 and FG 24 from SBF (Jerjen et al. 1998) have systematically lower distances, which is why we corrected their original SBF distance to NGC 59, $D=4.4 \mathrm{Mpc}$, by $+0.9 \mathrm{Mpc}$.

The distribution of the 21 galaxies from Table 2 is presented in Fig. 3 in equatorial coordinates. Three of the brightest spiral galaxies are shown as filled squares, and dIrr and dSph galaxies are shown as filled and open circles, respectively. Radial velocities of the galaxies with respect to the LG centroid are indicated by numbers. As can be seen, the distribution of galaxies in Sculptor exhibits the absence of any distinct center, and it does not show a sharp boundary either. Most of the galaxies are situated at low supergalactic latitudes, $|\mathrm{SGB}|<10^{\circ}$, where projection effects makes it difficult to restore the 3-D structure of the group. Referring to Table 3, we recognize that the spiral galaxy NGC 253 surpasses all other galaxies in this group by more than a factor of five in luminosity. Therefore, we consider NGC 253 as the dynamical center of the Sculptor group.

Puche \& Carignan (1988) estimated the distance to NGC 253 and some other galaxies in Sculptor, based on the
Tully-Fisher relation. However, for its calibration, they used old data on galaxy distances. A revised relation "absolute magnitude - H I line width" for 6 galaxies in Sculptor with new distance estimates is presented in Fig. 4. Here, the line width is corrected for galaxy inclination to the line of sight. Using the regression line, $M_{B}=-7.0 \log \left(W_{\mathrm{c}}\right)-1.8$, we estimated the distances to five galaxies: NGC 55, NGC 247, NGC 625, ESO 349-031, and ESO 149-03, presented in Col. 8 of Table 2 .

The Hubble diagram showing velocity versus distance for 20 galaxies in Sculptor is given in Fig. 5. Galaxies with accurate distance estimates ("Cep", "RGB"”, "SBF") are shown as filled circles, while galaxies with distances from the T-F relation are indicated by crosses, and the brightest spiral galaxy NGC 253 is shown as a square. Four dSph galaxies without velocities are shown conditionally by vertical bars. The solid line corresponds to the Hubble parameter $H=75 \mathrm{~km} \mathrm{~s}^{-1} \mathrm{Mpc}^{-1}$. At small distances the line deviates from a straight line due to a decelerating gravitational action of the Local Group, the total mass of which is adopted to be $M_{\mathrm{LG}}=1.3 \times 10^{12} M_{\odot}$ (Karachentsev et al. 2002).

Based on the data in Figs. 3 and 5, we can describe the structure of the galaxy complex in Sculptor in the following way.

a) Three galaxies with $V_{\mathrm{LG}}>400 \mathrm{~km} \mathrm{~s}^{-1}$ (ESO 149-03, NGC 59, and DDO 226) apparently are background objects. 
Table 3. Properties of nearby groups in Sculptor.

\begin{tabular}{|c|c|c|c|c|c|c|c|c|}
\hline Group & $N$ & $\begin{array}{c}D \\
\mathrm{Mpc}\end{array}$ & $\begin{array}{c}<R_{\mathrm{p}}> \\
\mathrm{kpc}\end{array}$ & $\begin{array}{c}\sigma_{V} \\
\mathrm{~km} \mathrm{~s}^{-1}\end{array}$ & $\begin{array}{c}L_{B} \\
10^{10} L_{\odot}\end{array}$ & $\begin{array}{c}M_{\mathrm{vir}} / L_{B} \\
M_{\odot} / L_{\odot}\end{array}$ & $\begin{array}{c}M_{\text {orb }} / L_{B} \\
M_{\odot} / L_{\odot}\end{array}$ & $\begin{array}{l}T_{\text {cross }} \\
\text { Gyr }\end{array}$ \\
\hline $\begin{array}{l}\text { N300, N55, } \\
\text { E294-10, } \\
\text { E410-05 }\end{array}$ & 4 & 1.95 & 279 & 15 & 0.33 & 54 & 45 & 18.6 \\
\hline $\begin{array}{l}\text { N253, N247, } \\
\text { DDO6, Sc22, } \\
\text { KDG2, FG24 }\end{array}$ & 6 & 3.94 & 370 & 54 & 1.91 & 143 & 83 & 6.9 \\
\hline $\begin{array}{l}\text { N7793, UA442, } \\
\text { E349-31 }\end{array}$ & 3 & 3.91 & 205 & 34 & 0.30 & 260 & 140 & 6.0 \\
\hline
\end{tabular}

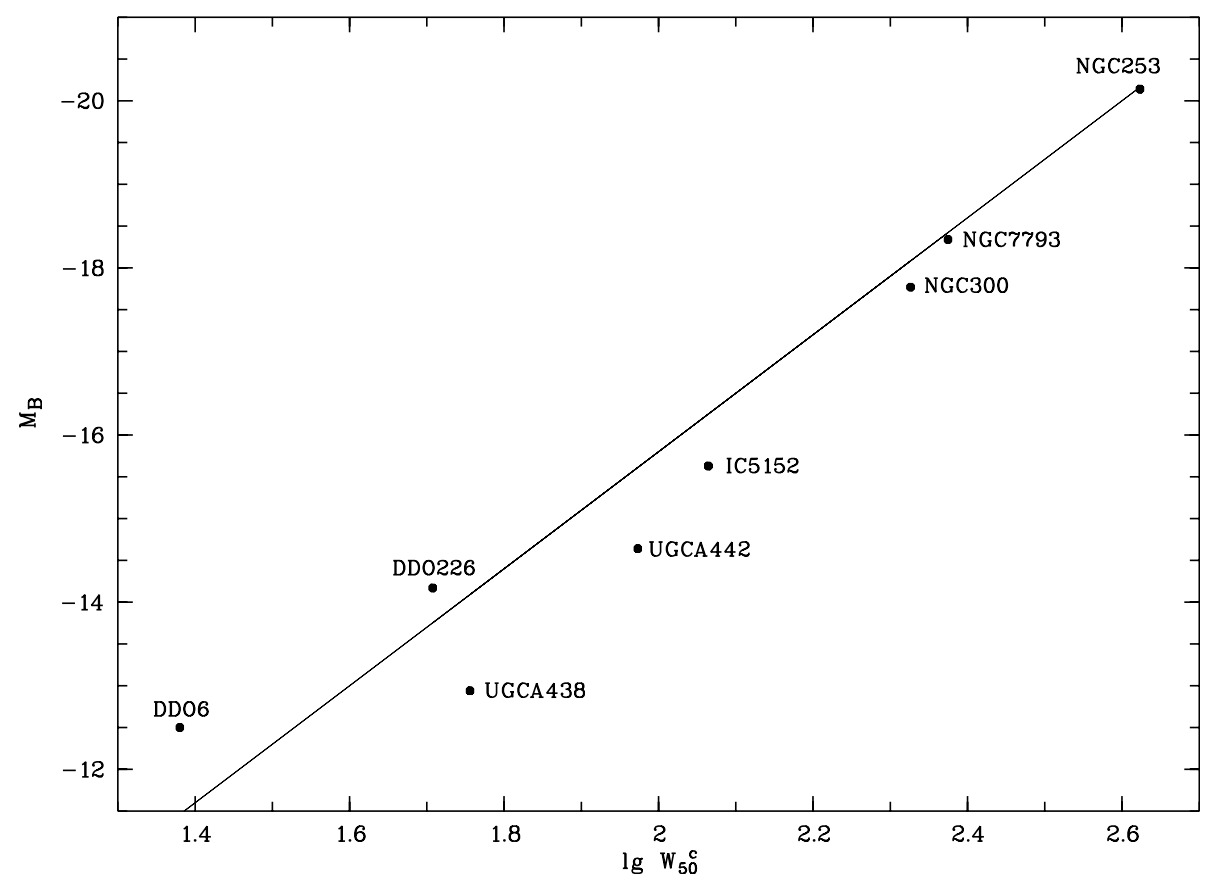

Fig. 4. Tully-Fisher relation for eight galaxies in Sculptor with accurate distances.

NGC 59 and DDO 226 probably form a wide pair with a linear projected distance of $580 \mathrm{kpc}$ and a radial velocity difference of $13 \mathrm{~km} \mathrm{~s}^{-1}$.

b) In front of the complex there is a pair of bright galaxies, NGC 300 and NGC 55, with two dSph companions, ESO 410-05 and ESO 294-10. The mean distance to this loose quartet is $1.95 \mathrm{Mpc}$. This is only $0.6 \mathrm{Mpc}$ more distant than to another known loose quartet: NGC 3109, Sex A, Sex B, and Antlia, located at the LG edge. Possibly, two other dIrr galaxies, UGCA 438 and IC 5152, are associated with the NGC 300 group, too.

c) The brightest spiral galaxy NGC 253 together with its companions, NGC 247, DDO 6, Sc 22, KDG 2 and FG 24, can be considered as the Sculptor complex' core. The last three $\mathrm{dSph}$ galaxies do not have radial velocities so far. Apart from the NGC 253 group, there is a galaxy triplet, NGC 7793, UGCA 442, and ESO 349-031. The radial distances of NGC 253 and NGC 7793 are almost the same within the measurement errors.

d) Among the remaining galaxies, NGC 625 and ESO 245-05 have an angular separation of 2.9 and a radial velocity difference of only $27 \mathrm{~km} \mathrm{~s}^{-1}$. However, their distance estimates differ significantly. Because NGC 625 deviates essentially from the Hubble regression line in Fig. 5, we assume its T-F distance to be underestimated by approximately $2 \mathrm{Mpc}$. This assumption can be easily proven by measuring the TRGB distance to this poorly studied galaxy.

The total mass of each group can be estimated from the virial balance of kinetic and potential energies (Limber \& Mathews 1960),

$M_{\mathrm{vir}}=3 \pi N(N-1)^{-1} G^{-1} \sigma_{\mathrm{v}}^{2} R_{\mathrm{H}}$,

where $N$ is the number of galaxies in the group, $\sigma_{\mathrm{v}}^{2}$ is the radial velocity dispersion, $R_{\mathrm{H}}$ is the mean projected harmonic 


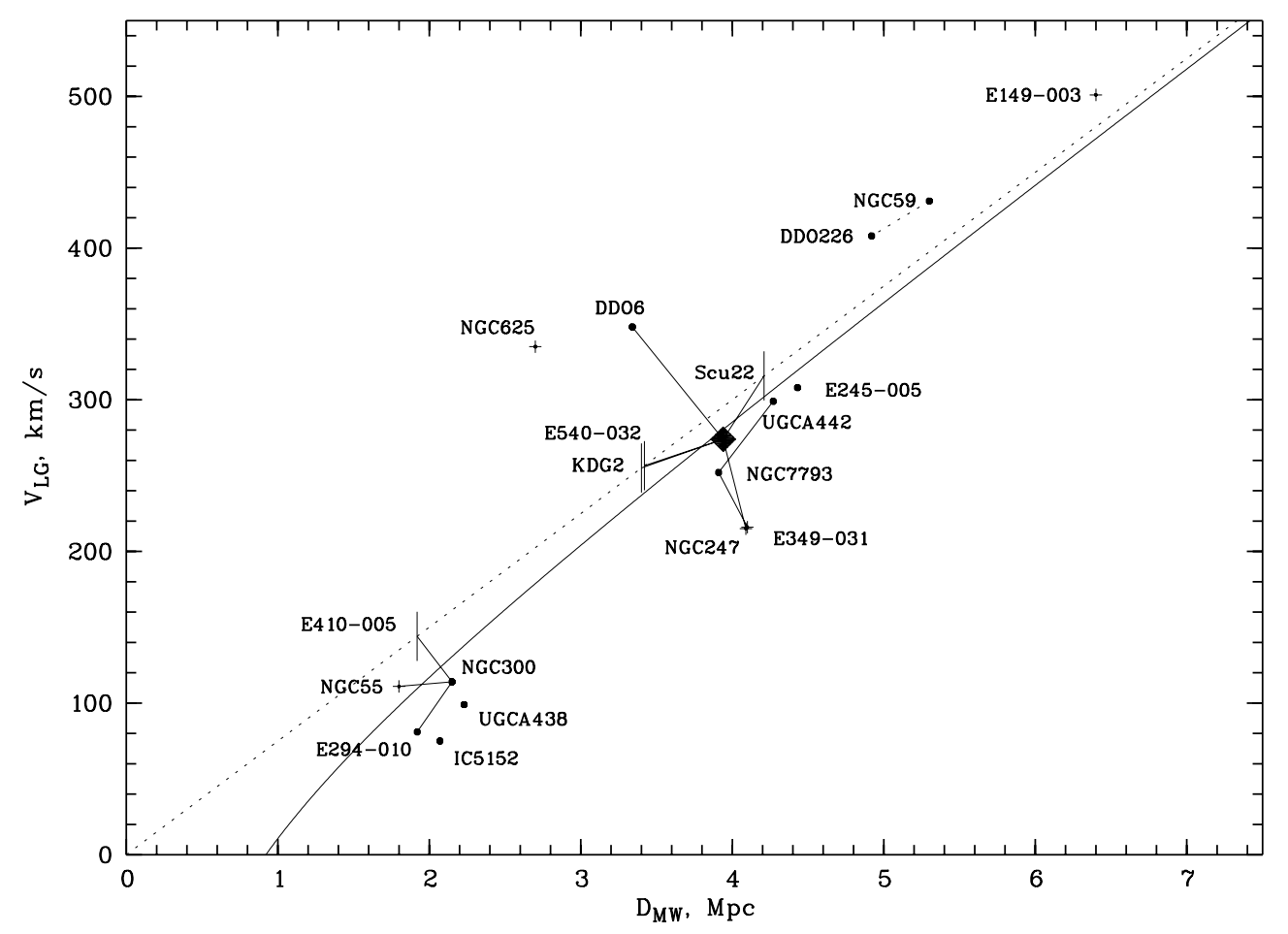

Fig. 5. Radial velocity-distance relation for 20 nearby galaxies in Sculptor. The galaxies with accurate distance estimates are shown as filled circles, and galaxies with T-F distances are shown as crosses. Four dSph galaxies without radial velocities are indicated by vertical bars. The brightest galaxy, NGC 253, is indicated by a square. The solid line corresponds to the Hubble relation with $H=75 \mathrm{~km} \mathrm{~s}^{-1} \mathrm{Mpc}^{-1}$, curved at small distances assuming a decelerating gravitational action of the Local Group with a total mass of $1.3 \times 10^{12} M_{\odot}$. Probable companions of the most luminous galaxies are connected with these by straight lines.

radius, and $G$ is the gravitational constant. Such an approach assumes that the characteristic crossing time of the group, $T_{\text {cross }}=\left\langle R_{p}\right\rangle / \sigma_{\mathrm{v}}$, is low in comparison with the age of the Universe (here $\left\langle R_{\mathrm{p}}\right\rangle$ means the average projected radius of the group).

Another way to estimate the total mass of group was proposed by Bahcall \& Tremaine (1981). Assuming the motions of dwarf galaxies around the main group member to be closed Keplerian motions with orbit eccentricity $e$, in the case of random orientation of galaxy orbits we obtain

$M_{\mathrm{orb}}=(32 / 3 \pi) G^{-1}\left(1-2 e^{2} / 3\right)^{-1}\left\langle R_{\mathrm{p}} \Delta V_{\mathrm{r}}^{2}\right\rangle$,

where $R_{\mathrm{p}}$ and $\Delta V$ are projected distance and radial velocity of a companion with respect to the main group member.

The basic dynamical parameters of the three mentioned groups in Sculptor are presented in Table 3. In the case of orbital mass estimates the mean eccentricity $e=0.7$ is adopted. As seen from these data, the virial/orbital mass-to-luminosity ratios of the groups lie in the range of 45 to $260 M_{\odot} / L_{\odot}$. However, for all the groups their crossing time, 6-18 Gyr, is comparable to the time of cosmic expansion, $1 / H_{0}$, which makes the derived mass estimates too unreliable.

According to Lynden-Bell (1981) and Sandage (1986), in the expanding universe any dense enough group with a total mass $M_{0}$ may be characterized by a "zero-velocity surface", which separates the group from the Hubble flow. In the case of spherical symmetry, the radius of this surface, $R_{0}$, is expressed via the total mass of the group and the Hubble constant, $H_{0}$, by a simple relation

$M_{0}=\left(\pi^{2} / 8 G\right) H_{0}^{2} R_{0}^{3}$.

For estimating $R_{0}$, we calculated for any galaxy with the distance $D$ and radial velocity $V$ its spatial separation from NGC 253

$R^{2}=D^{2}+D_{N 253}^{2}-2 D D_{N 253} \cos \theta$

and its projected radial velocity with respect to NGC 253

$\left(V-V_{N 253}\right)_{\mathrm{p}}=V \cos \lambda-V_{N 253} \cos (\theta+\lambda)$,

where $\tan \lambda=D_{N 253} \sin \theta /\left(D-D_{N 253} \cos \theta\right)$. Here we assumed that the peculiar velocities of the galaxies are small in comparison with velocities of the regular Hubble flow. The estimated values $\left(V-V_{N 253}\right)_{\mathrm{p}}$ and $R$ for 15 galaxies around NGC 253 are presented in Col. 9 of Table 2. The distribution of relative radial velocities and spatial separations is shown in Fig. 6. Here galaxies with accurate ("Cep", "RGB", "SBF") and with rough ("TF") distance estimates are indicated by filled circles and crosses, respectively. As seen from Fig. 6, all galaxies outside $R=0.7 \mathrm{Mpc}$ (in particular, NGC 7793 together with its companions) move away from NGC 253. Based on these (still incomplete) data, we can conclude that the radius of the zero-velocity surface for the NGC 253 group is $R_{0}=0.7 \pm$ $0.1 \mathrm{Mpc}$. According to Eq. (3) this corresponds to a total mass of $M_{0}=(0.55 \pm 0.22) \times 10^{12} M_{\odot}$ or $M_{0} / L_{B}=(29 \pm 11) M_{\odot} / L_{\odot}$, which is $3-5$ times lower than the virial/orbital mass estimates 


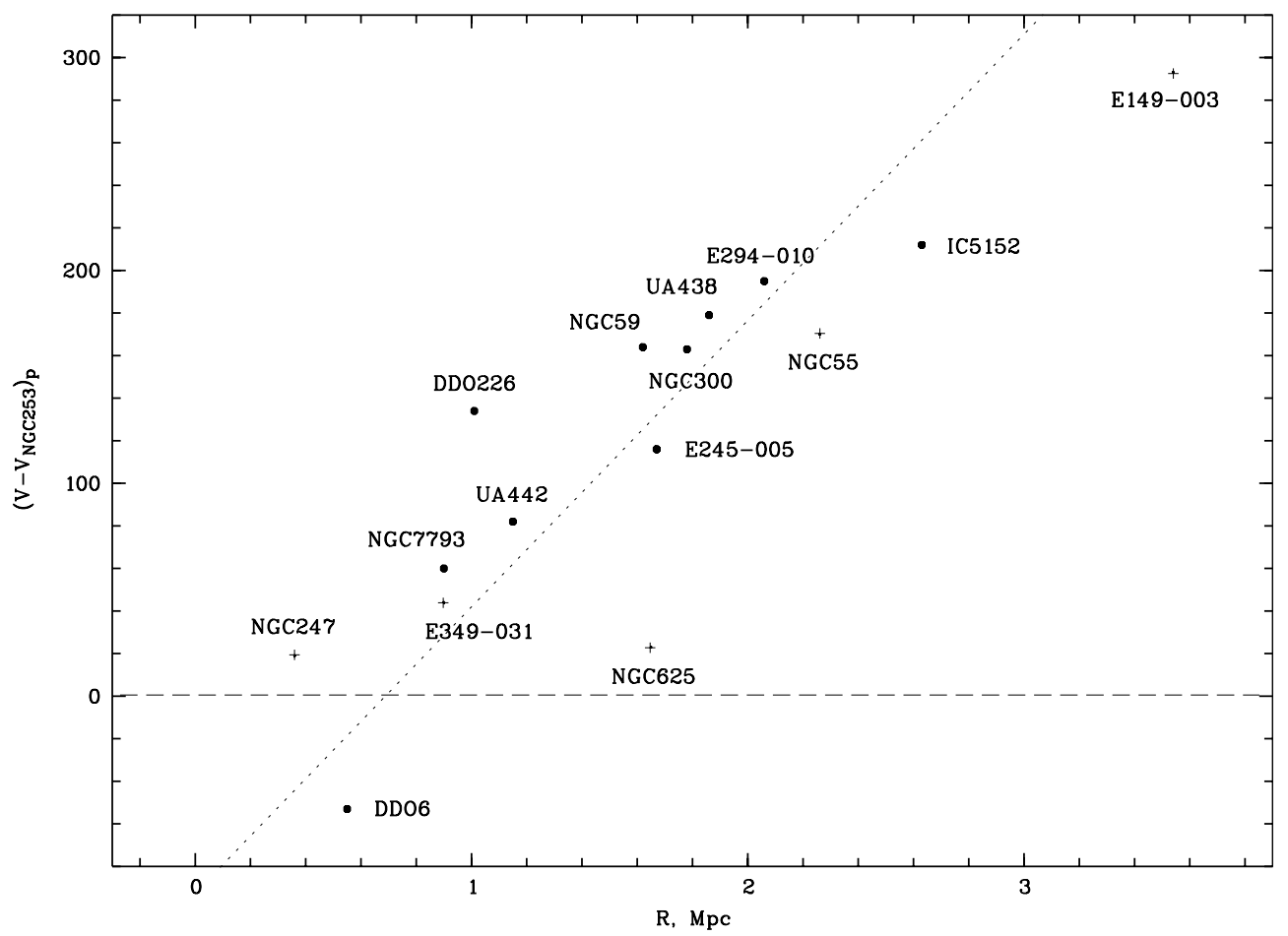

Fig. 6. The distribution of the radial velocity difference and of the space distance of nearby galaxies with respect to NGC 253 . These data yield a radius of the zero-velocity surface of $R_{0}=0.7 \mathrm{Mpc}$.

derived above. Measurements of radial velocities for the remaining four dSph galaxies, situated in the range $R=0.47-$ $2.07 \mathrm{Mpc}$, will allow one to derive the radius $R_{0}$ and the total mass of the NGC 253 group with higher accuracy.

\section{Concluding remarks}

Measurements of accurate distances to 9 galaxies in Sculptor led to essential clarifying of the structure and kinematics of this nearby complex of galaxies. However, nine other presumably nearby galaxies in the same area (with $V_{\mathrm{LG}}<500 \mathrm{~km} \mathrm{~s}^{-1}$ or $D<5 \mathrm{Mpc}$ ) still remain without reliable distance estimates (NGC 55, NGC 625, NGC 247, SDIG, and ESO 149-03) or radial velocity estimates (ESO 410-05, KDG 2, FG 24, and Sc 22). Our new data on galaxy distances confirm the conclusion drawn by Jerjen et al. (1998) that the so-called group in Sculptor is a loose "cloud" of galaxies of $1 \times 6 \mathrm{Mpc}$ in size, extended along the line of sight. Apparently, the near and the far parts of this "cigar" are not gravitationally bound each other, but take part in the general Hubble flow. In this sense, the Sculptor cloud looks like another nearby cloud, Canes Venatici I (Karachentsev et al. 2003b). Both these loose clouds are populated mostly by dwarf galaxies, and their luminosity function has a flat "primordial" shape. As seen from Fig. 7 in Karachentsev et al. (2003a), the Galaxy complexes in Sculptor and Canes Venatici together with the Local Group form a filament extending over $\sim 10 \mathrm{Mpc}$.

The nearby galaxy complex in Sculptor seems to us a suitable case to study the "anatomy" of virial mass excess. In projection onto the sky, the Sculptor filament has a rather high overdensity, and can be easely identified as a usual group by the "friend of friends" algorithm (Huchra \& Geller 1982) or by the method of hierarchical trees (Materne 1978). In his Nearby Galaxy Catalog, Tully (1988) denotes the Sculptor group by the number "14-13". Based on radial velocities and projected separations of 11 members of the group, Tully (1987) estimated its virial mass-to-luminosity ratio to be $M_{\text {vir }} / L_{B}=$ $328 M_{\odot} / L_{\odot}$. There the galaxies NGC 55, NGC 253, NGC 7793, and DDO 226 were considered as members of a single group. Apart from real and probable members of the Sculptor complex, Tully included in the group also the galaxy PGC 71145, whose radial velocity is $+16 \mathrm{~km} \mathrm{~s}^{-1}$ (instead of $+1600 \mathrm{~km} \mathrm{~s}^{-1}$ as a result of a misprint in Longmore et al. 1982). Comparing the ratio $M_{\text {vir }} / L_{B}$ from Tully (1987) with our estimate of the total mass-to-luminosity ratio, $29 \pm 11 M_{\odot} / L_{\odot}$, we conclude that the usage of the more reliable and precise observational data that are now available, as well as our new approach to the determination of mass can decrease the total mass estimate of the group by one order of magnitude.

Acknowledgements. Support for this work was provided by NASA through grant GO-08601.01-A from the Space Telescope Science Institute, which is operated by the Association of Universities for Research in Astronomy, Inc., under NASA contract NAS5-26555. This work was partially supported by RFBR grant $01-02-16001$ and DFG-RFBR grant 02-02-04012. D.G. gratefully acknowledges support from the Chile Centro de Astrofísica FONDAP No. 15010003.

The Digitized Sky Surveys were produced at the Space Telescope Science Institute under U.S. Government grant NAG W-2166. The images of these surveys are based on photographic data obtained using the Oschin Schmidt Telescope on the Palomar Mountain and the UK Schmidt Telescope. The plates were processed into the present compressed digital form with permission of these institutions. 
This project made use of the NASA/IPAC Extragalactic Database (NED), which is operated by the Jet Propulsion Laboratory, Caltech, under contract with the National Aeronautics and Space Administration.

\section{References}

Bahcall, J. N., \& Tremaine, S. 1981, ApJ, 244, 805

Barnes, D. G., Staveley-Smith, L., \& de Blok, W. J. 2001, MNRAS, 322,486

Côte, S., Freeman, K. C., Carignan, C., \& Quinn, P. J. 1997, AJ, 114, 1313

Bellazzini, M., Ferraro, F. R., \& Pancino, E. 2001, ApJ, 556, 635

Da Costa, G. S., \& Armandroff, T. E. 1990, AJ, 100, 162

de Blok, W. J., Zwaan, M. A., Dijkstra, M., et al. 2002 [astro-ph/0111238]

Dolphin, A. E. 2000a, PASP, 112, 1383

Dolphin, A. E. 2000b, PASP, 112, 1397

Feitzinger, J. W., \& Galinski, T. 1985, A\&AS, 61, 503

Ferrarese, L., Mould, J. R., Kennicutt, R. C. Jr., et al. 2000, ApJ, 529, 745

Freedman, W., Madore, B. F., Hawley, S. L., et al. 1992, ApJ, 396, 80

Grebel, E. K., Seitzer, P., Dolphin, A., et al. 2000, in Stars, Gas, and Dust in Galaxies: Exploring the Links, ed. D. Alloin, K. Olsen, \& G. Galaz (Provo: ASP), ASP Conf. Ser., 221, 147

Huchra, J. P., \& Geller, M. J. 1982, ApJ, 257, 423

Huchtmeier, W. K., Karachentsev, I. D., Karachentseva, V. E, \& Ehle, M. 2000, A\&AS, 141, 469

Jerjen, H., \& Rejkuba, M. 2001, A\&A, 371, 487

Jerjen, H., Freeman, K. C., \& Binggeli, B. 2000, AJ, 119, 166

Jerjen, H., Freeman, K. C., \& Binggeli, B. 1998, AJ, 116, 2873

Karachentsev, I., \& Makarov, D. 1996, AJ, 111, 535

Karachentsev, I. D., Sharina, M. E., Grebel, E. K., et al. 2000, ApJ, 542,128

Karachentsev, I. D., Sharina, M. E., Makarov, D. I., et al. 2002, A\&A, 389,812
Karachentsev, I. D., Makarov, D. I., Sharina, M. E., et al. 2003a, A\&A, 398, 479

Karachentsev, I. D., Sharina, M. E., Grebel, E. K., et al. 2003b, A\&A, 398, 467

Karachentseva, V. E., \& Karachentsev, I. D. 2000, A\&AS, 146, 359

Karachentseva, V. E., \& Karachentsev, I. D. 1998, A\&AS, 127, 409

Lauberts, A. 1982, ESO/Uppsala Survey of ESO(B) Atlas (Garching: ESO)

Lee, M. G., Freedman, W. L., \& Madore, B. F. 1993, ApJ, 417, 553

Limber, D. N., \& Mathews, W. G. 1960, ApJ, 132, 286

Longmore, A. J., Hawarden, T. G., Mebold, U., et al. 1982, MNRAS, 200,325

Lynden-Bell, D. 1981, Observatory, 101, 111

Materne, J. 1978, A\&A, 63, 401

Mendez, B., Davis, M., Moustakas, J., et al. 2002, AJ, 124, 213

Nilsen, P. 1974, Uppsala Astr. Observ. report, 5, 1

Paturel, G., Bottinelli, L., Di Nella, H., et al. 1996, Catalogue of Principal Galaxies, (LEDA), Saint-Genis Laval, Observatoire de Lyon

Puche, D., \& Carignan, C. 1988, AJ, 95, 1025

Sakai, S., \& Madore, B. F. 1999, ApJ, 526, 599

Sakai, S., Madore, B. F., \& Freedman, W. L. 1996, ApJ, 461, 713

Salaris, M., \& Cassisi, S. 1997, MNRAS, 289, 406

Sandage, A. 1986, ApJ, 307, 1

Schlegel, D. J., Finkbeiner, D. P., \& Davis, M. 1998, ApJ, 500, 525

Seitzer, P., Grebel, E. K., Dolphin, A. E., et al. 1999, BAAS

Staveley-Smith, L., Yuraszek, S., Koribalski, B. S., et al. 1998, AJ, 116,2717

Tully, R. B. 1987, ApJ, 321, 280

Tully, R. B. 1988, Nearby Galaxy Catalog (Cambridge Univ. Press)

Udalski, A., Wyrzykowski, L., Pietrzynski, G., et al. 2001, Acta Astronomica, 51, 221

van den Bergh, S. 1959, Publ. David Dunlap Obs., II, No. 5

Whiting, A. B., Hau, G. K., \& Irwin, M. 2002, ApJS, 141, 123 